TecnoLógicas

ISSN-p 0123-7799

ISSN-e 2256-5337

Vol. 21, No. 41, pp. 149-157

Enero-abril de 2018

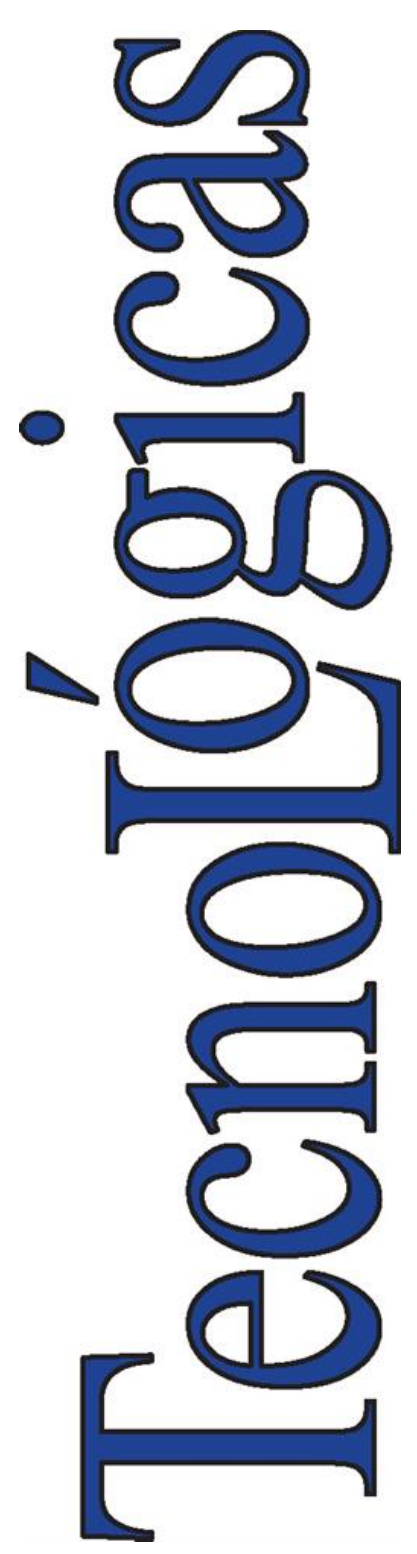

(C) Copyright 2015 por autores y Tecno Lógicas Este trabajo está licenciado bajo una Licencia Internacional Creative Commons Atribución (CC BY)

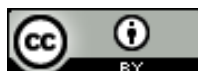

\section{Desarrollo y evaluación de un sistema interactivo para personas con discapacidad visual}

\section{Development and Evaluation of an Interactive System for the visually impaired}

\author{
Alma L. Esparza-Maldonado ${ }^{1}$, Lourdes Y. Margain- \\ Fuentes $^{2}$, Francisco J. Álvarez-Rodríguez ${ }^{3}$ \\ y Edgard I. Benítez-Guerrero ${ }^{4}$
}

Recibido: 08 de octubre de 2017

Aceptado: 04 de diciembre de 2017

Cómo citar / How to cite

A. L. Esparza-Maldonado, L. Y. Margain-Fuentes, F. J. ÁlvarezRodríguez y E. I. Benítez-Guerrero, Desarrollo y evaluación de un Sistema Interactivo para personas con discapacidad visual. TecnoLógicas, vol. 21, no. 41, pp. 149-157, 2018.

1 MSc en Ciencias, Ciencias de la Computación, Universidad Veracruzana, Xalapa-México, alma2_laura5@hotmail.com

$2 \mathrm{PhD}$ en Ciencias de la Computación, Sistemas Estratégicos de Información, Universidad Politécnica de Aguascalientes, Aguascalientes-México, lourdes.margain@upa.edu.mx

$3 \mathrm{PhD}$ en Ingeniería, Ciencias de la computación, Universidad Autónoma de Aguascalientes, Aguascalientes-México, fjalvar@correo.uaa.mx

$4 \mathrm{PhD}$ en Informática, Ciencias de la computación, Universidad Veracruzana, Xalapa, Veracruz, edbenitez@uv.mx 


\title{
Resumen
}

Lo que se presenta en este documento es una experimentación sobre el desarrollo y los resultados de las pruebas realizadas de una aplicación para computadora, enfocadas a personas con discapacidad visual. Los objetivos del sistema interactivo son: dar a conocer algunos lugares del estado de Aguascalientes, México; y fortalecer la memoria del usuario ciego. El propósito de este artículo es que mediante la construcción del sistema y las pruebas realizadas se da a conocer el grado de interactividad para personas ciegas. El construir aplicaciones con calidad enfocada a la interactividad es importante, ya que, para personas con discapacidad, puede mejorar el grado de uso de las tecnologías (dispositivos móviles). Para determinar la calidad es necesario conocer las características que debe de tener este tipo de aplicaciones, como lo son: pantalla grande, teclado adecuado, ergonomía, entre otras [1], dependiendo del tipo de discapacidad al que se esté refiriendo, es decir, se debe de caracterizar la aplicación para poder determinar cómo desarrollar la aplicación y la medición de la calidad mediante el grado de interacción obtenido. La principal aportación de este trabajo es mostrar los resultados obtenidos del grado de interactividad del sistema al ser utilizado por personas ciegas, así como mostrar parte del proceso de producción de software en el trabajo en equipos definido por Watts Humphrey utilizado para la construcción de la aplicación (TSP) [2].

\section{Palabras clave}

Discapacidad visual, aplicaciones para personas ciegas, sistemas interactivos para personas con discapacidad, proceso de desarrollo con calidad, características de sistema interactivo para discapacidad.

\begin{abstract}
This document presents the experimentation, development and results of testing conducted on a computer application designed for visually impaired people. The interactive system aims to promote some places in the State of Aguascalientes, Mexico, and improve the memory of blind users. The purpose of this article is to describe the construction of the system and the testing conducted to measure the degree of interactivity with blind people. Creating interactivity-focused applications is important since it can improve the degree of technology usage (mobile devices) of people with disabilities. To assess the quality of this type of software, the specifications they should meet need to be established. They involve big screens, adapted keyboards and ergonomics [1] and depend on the type of disability being addressed. Therefore, they should be characterized to determine how to develop them and assess their quality with the degree of interaction obtained. The main contribution of this work is presenting the results of the degree of interactivity of the system when used by blind people and detailing part of the Team Software Process (TSP) defined by Watts Humphrey and adopted for developing the application [2].
\end{abstract}

\section{Keywords}

Visual impairment, Applications for blind people, Interactive systems for people with disabilities, Quality development process, Features of accessible interactive systems. 


\section{INTRODUCCIÓN}

La discapacidad se refiere a las personas que tienen una o más deficiencias, ya sean físicas, mentales, intelectuales o sensoriales [3] y que al interactuar con distintos ambientes del entorno social pueden impedir su participación plena y efectiva en igualdad de condiciones. Koon dice que alrededor del $15 \%$ de la población mundial se encuentra afectada por algún tipo de discapacidad [4]. En el año 2010, las personas que presentaron algún tipo de discapacidad en México sobrepasaron los $5 \mathrm{mi}$ llones, lo cual representa el $5.1 \%$ de la población [5].

Los seres humanos, ya sea con algún tipo de discapacidad o sin ella, nacen libres e iguales en dignidad y en derechos, hoy en día aún se violan derechos de las personas con algún tipo de discapacidad, por lo que se está tratando de resolver esta problemática con la inclusión de las personas con discapacidad a diferentes contextos [6]. Un hecho importante es que las tecnologías se utilizan en diversos sectores de la sociedad y así mismo afectan a diferentes ámbitos de las ciencias humanas [7].

La inclusión de las personas con discapacidad en el uso de las tecnologías de información es un aspecto importante, dado que diferentes aplicaciones interactivas requieren ser desarrolladas o adecuadas acorde con las necesidades y características de cada discapacidad, por ejemplo, considerar que la interfaz sea amigable y fácil de usar.

Drummond dice: "un sistema interactivo tiene el potencial para la variación y la imprevisibilidad en su respuesta, y dependiendo del contexto bien puede ser considerado más en términos de una composición o improvisación estructurada que en lugar de un instrumento" [8].

En el proceso de desarrollo de software se utilizan diferentes modelos para definir un conjunto de actividades, métodos, prácticas y modificaciones, que los desarrolladores usan al construir y mantener softwa- re, así como sus productos asociados [9]. El modelo de proceso de software es una representación abstracta de un proceso. Los procesos del modelo, pueden incluir un conjunto de actividades, las cuales son parte del mismo proceso, los productos de software, objetivos, responsabilidades, subprocesos [10] y los roles que están involucrados en la ingeniería del software. Un proceso para el desarrollo de software es el conocido como Team Software Process (TSP).

El término Team Software Process se refiere a una metodología que es utilizada en la construcción de aplicaciones a nivel de equipo para la mejora de procesos [2]. La estructura del TSP utiliza múltiples ciclos de desarrollo para construir un producto final.

La calidad del software se refiere al cumplimiento de los requerimientos funcionales explícitos o implícitos y el rendimiento en el producto con la documentación de los estándares previamente definidos [11], en otras palabras, la calidad se fundamenta en que el consumidor se encuentre satisfecho con el producto a un precio accesible.

En el presente documento se describe del desarrollo de una aplicación para personas ciegas la cual es llamada "Conociendo mi cuidad", esta aplicación es de escritorio, desarrollada en la plataforma de Visual Studio. Las primeras pruebas realizadas consistieron en aplicar encuestas a los usuarios principales que son seis personas ciegas adultas, las cuales evalúan el grado de interacción en los diferentes componentes. En pruebas posteriores serán realizadas por especialistas en temas de usabilidad.

\section{METOdOLOGía}

La aplicación desarrollada es un juego de memoria para personas ciegas que persigue dos objetivos: el primero es que el usuario ciego conozca los principales luga- 
res turísticos del estado de Aguascalientes, así como comidas tradicionales y arquitectura, para que en un futuro pueda desenvolverse como guía turístico o encuentre un trabajo y se le facilite el ubicarse dentro del estado de Aguascalientes; el segundo objetivo es que el usuario desarrolle habilidades de memoria y de esta forma recordar ubicaciones y características del primer objetivo.

Para el desarrollo de la aplicación se utilizó la metodología TSP [2]. Este se encuentra entre las metodologías enfocadas a la producción de software con calidad; enfocándose en el equipo de trabajo en el cual se asignan los roles, líder de equipo, análisis y diseño, desarrollador, soporte y calidad, a cada integrante del equipo.

El equipo de desarrollo de esta aplicación se encuentra conformado por estudiantes de la carrera de Ingeniería en Sistemas Estratégicos de Información (ISEI), de la Universidad Politécnica de Aguascalientes.

Para la construcción de la aplicación deben considerarse características específicas que debe cumplir un sistema interactivo, según la discapacidad a la que se esté centrando el desarrollo. Las características que se deben de considerar para una aplicación, dependiendo el tipo de discapacidad, son el tamaño de los botones, sonido, ergonomía, entre otras, como se puede observar en la Tabla 1. La aplicación se encuentra actualmente instalada en las computadoras del Sistema Nacional para el Desarrollo Integral de la Familia, conocido como DIF estatal de Aguascalientes, la cual es una institución que se enfoca en desarrollar el bienestar de las familias y personas con discapacidad.

En la construcción de la aplicación interactiva se requiere la asignación de roles al equipo de desarrollo, sin embargo, en este trabajo solo se explican las actividades realizadas por el rol de administrador de análisis de requerimientos y diseño, el cual realiza el diseño de la aplicación con base a las características analizadas en la Tabla 1 [12]. Estas características de diseño son las que debe de considerarse para un usuario ciego. El diseño consta de botones que representan las tarjetas de un memorama, estos botones son de un tamaño grande, se diseñó un menú simple y el acceso a la aplicación es fácil, el diseño de la aplicación se puede observar en las Fig. 1 y 2 .

Tabla 1. Características que debe de considerar el sistema interactivo dependiendo de la discapacidad [12].

\begin{tabular}{|c|c|c|c|c|}
\hline \multirow{2}{*}{ Característica del sistema interactivo } & \multicolumn{4}{|c|}{ Discapacidad } \\
\hline & Visual & Auditiva & Cognitiva & Física \\
\hline $\begin{array}{l}\text { Pantalla } \\
\text { Grande } \\
\text { Contraste suficiente } \\
\text { Configurable }\end{array}$ & $\mathrm{X}$ & & $\mathrm{X}$ & $\mathrm{X}$ \\
\hline $\begin{array}{l}\text { Funciones y Menú } \\
\text { Mínimo número de funciones y menús } \\
\text { Debe ser fácil el acceso al menú }\end{array}$ & $\mathrm{X}$ & & & \\
\hline $\begin{array}{l}\text { Ergonomía } \\
\text { Tamaño adecuado de terminales, teclas adecuadas, } \\
\text { separadas y perceptibles al tacto }\end{array}$ & $\mathrm{X}$ & & $\mathrm{X}$ & $\mathrm{X}$ \\
\hline Lenguaje de signos & & $\mathrm{X}$ & & \\
\hline Traducción de voz a datos & & $\mathrm{X}$ & & \\
\hline
\end{tabular}




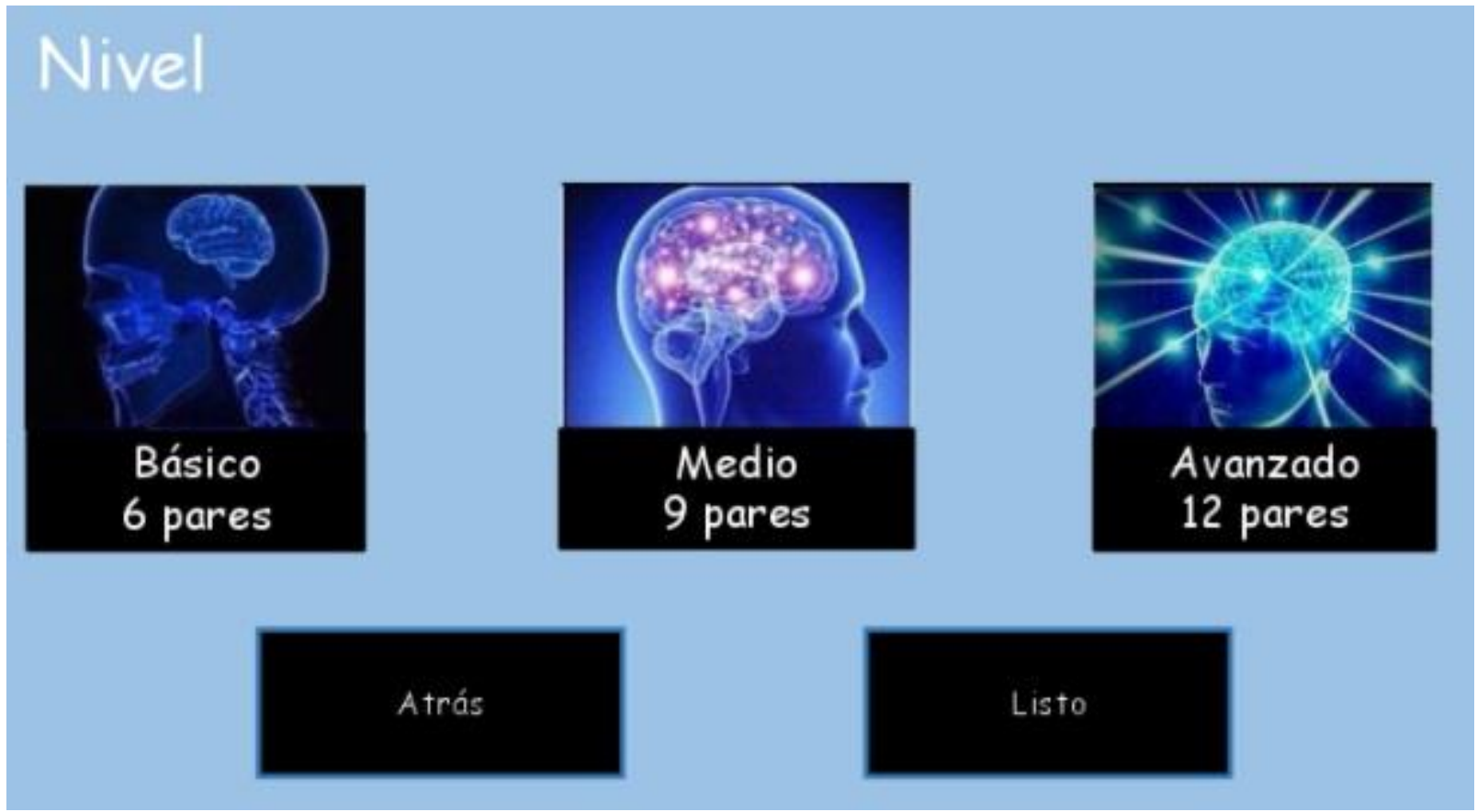

Fig. 1. Diseño del Menú del memorama para determinar el nivel del juego. Fuente: autores.

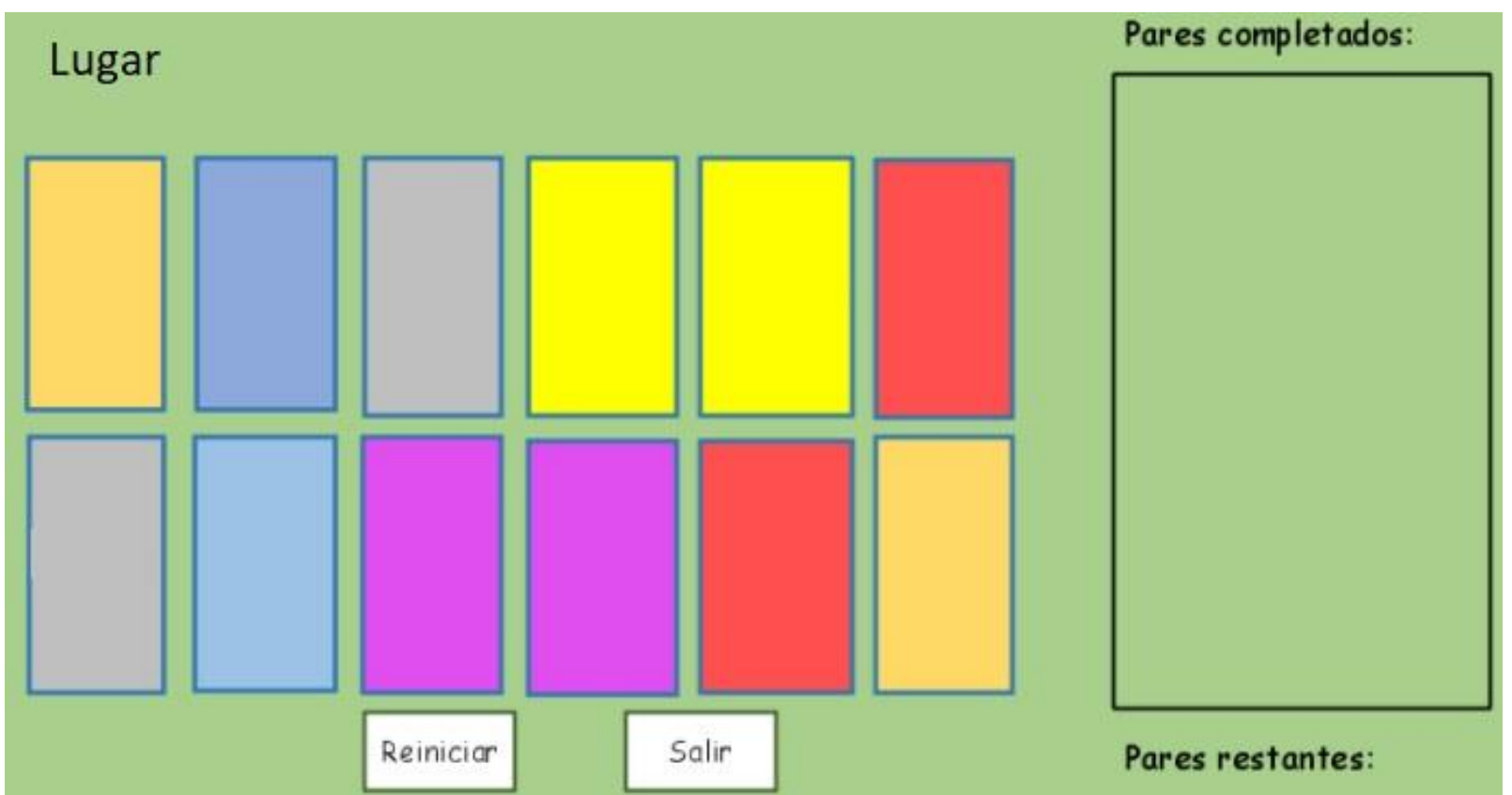

Fig. 2. Diseño de la aplicación del memorama para personas ciegas. Fuente: autores.

En la construcción a cada tarjeta se asignó una letra del teclado para recordar la tarjeta con la relación hacia la tecla presionada y encontrar el par de esta. $\mathrm{Al}$ encontrar un par, se da una breve explicación del lugar correspondiente al par encontrado mediante la reproducción de un audio. Además, cabe mencionar que la aplicación construida no requiere de un programa como el Jaws, el cual es un programa para computadora que lee en voz alta las actividades realizadas por el usuario, ya que la aplicación contiene instrucciones de audio para comenzar el juego.

Las pruebas realizadas se basaron en: Cañas y Waern [13], quienes plantearon 
una clasificación de la interacción entre los tipos informáticos y los factores humanos, el tercer nivel corresponde a los estilos de interacción de una sola persona con un sistema informático (sistema interactivo), dentro de los estilos de interacción se encuentran: línea de órdenes, menús y navegación, manipulación directa, interacción asistida [13]; y en Álvarez et al. [14], quien evaluó las tareas completadas por el usuario ciego, donde se mide el tiempo que tarda el usuario en completar dichas tareas en un sistema para personas con discapacidad visual y utilizando una encuesta. A continuación, se muestra los ítems de la encuesta.

¿Qué tan fácil es de usar la aplicación?

¿Qué tan intuitiva es la aplicación?

¿Qué tan confusa le resulta la aplicación?

¿Qué tantos problemas tienen con el acceso a los componentes?

¿Qué tan rápida es la aplicación?

¿Qué tan precisa es la aplicación en dar las instrucciones a seguir?

¿Qué tanto entiende las instrucciones a seguir?

¿Es fácil la utilización de la app?

¿Cuánto tiempo le llevó en aprender a utilizar la aplicación?

¿En qué nivel considera que la aplicación cumple su objetivo?

¿En qué nivel considera que la aplicación es útil?

¿Cuánto recomendaría la aplicación?

Para el uso de esta encuesta se utilizó una escala de 1 a 5 , siendo 1 totalmente en desacuerdo y 5 totalmente de acuerdo. Esta encuesta fue aplicada a seis personas ciegas, dos de ellas con ceguera de nacimiento y las otras cuatro personas con pérdida de visión en su edad adulta; cabe mencionar que todas las pruebas fueron aplicadas a personas adultas, la prueba solo se realizó a seis personas, ya que fueron las que accedieron a ser evaluadas, además Nielsen [15] menciona que evaluar a más de cinco usuarios la información se vuelve repetitiva y para medir la usabilidad se requiere mínimo 5 usuarios. Como nota y para aclarar la pregunta “¿Cuánto tiempo le llevó aprender a utilizar la aplicación?", se contesta con el tiempo y no basados en la escala.

La prueba fue realizada para determinar el grado del sistema interactivo "Conociendo mi ciudad", nombre dado por los desarrolladores de la aplicación.

\section{RESULTADOS Y DISCUSIONES}

En la Fig. 3 se puede observar un ejemplo del instrumento aplicado a un usuario ciego. Dicho instrumento fue aplicado a otras 5 personas ciegas para obtener resultados estadísticos, como se puede observar en la Fig. 4.

El objetivo del dato estadístico presentado en la Fig. 4 es mostrar los resultados, de la encuesta aplicada a los seis usuarios ciegos para determinar el grado de interacción del sistema. Como se puede observar, todos los usuarios recomiendan la aplicación, puesto que es fácil de utilizar, es iterativa con base a la definición de Drummond [8] para personas ciegas y cumple con el objetivo de la aplicación, el cual es ejercitar la memoria de la persona y el usuario reconozca los lugares representativos de la ciudad de Aguascalientes.

Como se puede observar en la gráfica, el nivel de satisfacción del usuario es aceptable, demostrando que el sistema es interactivo, solo existen 4 preguntas de las 12 en las que no existe una respuesta uniforme 
por parte de los seis usuarios, esto se debe al tipo de usuario que es, puesto que dos de ellos son ciegos desde su nacimiento y los otros cuatro perdieron o están perdiendo la vista en su edad adulta.
En la Fig. 5 se puede observar la utilización de la aplicación por uno de los usuarios, el cual tiene ceguera desde los tres años de edad; la prueba se realizó con cinco usuarios más.

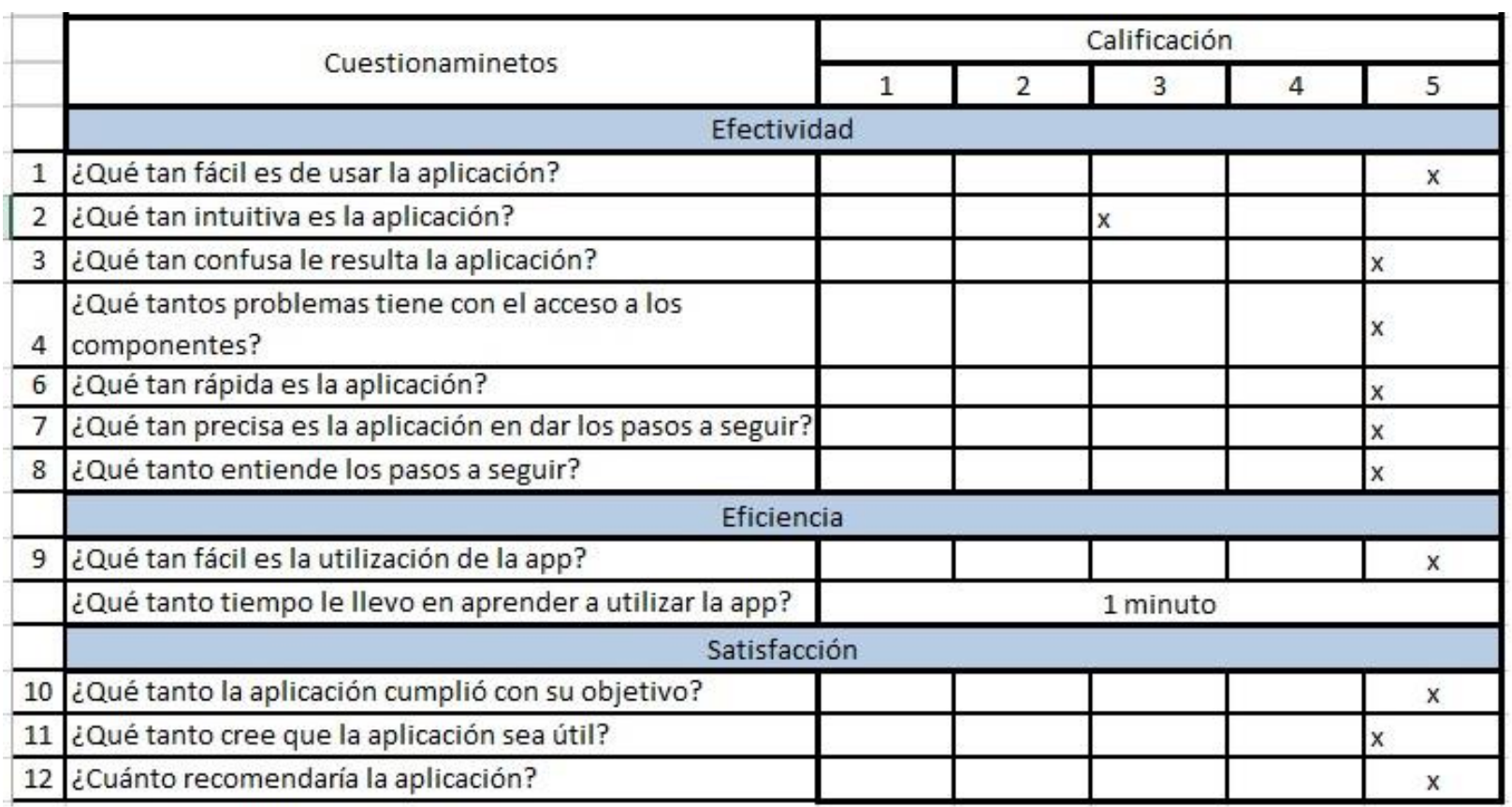

Fig. 3. Respuestas de usuario ciego a la encuesta aplicada. Fuente: Autores.

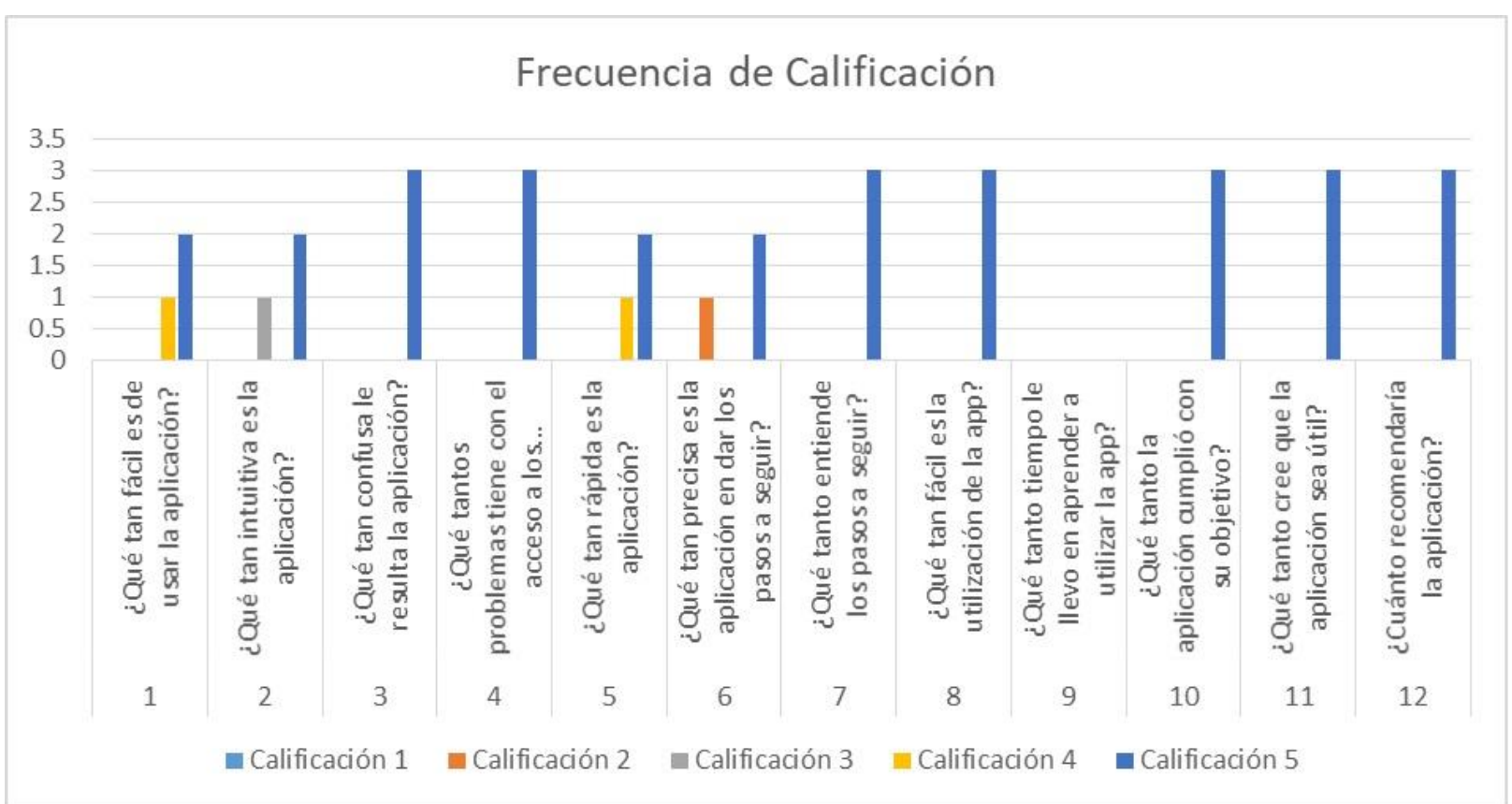

Fig. 4. Gráfica de la frecuencia obtenida según las respuestas dadas por los usuarios de la aplicación. Fuente: autores. 


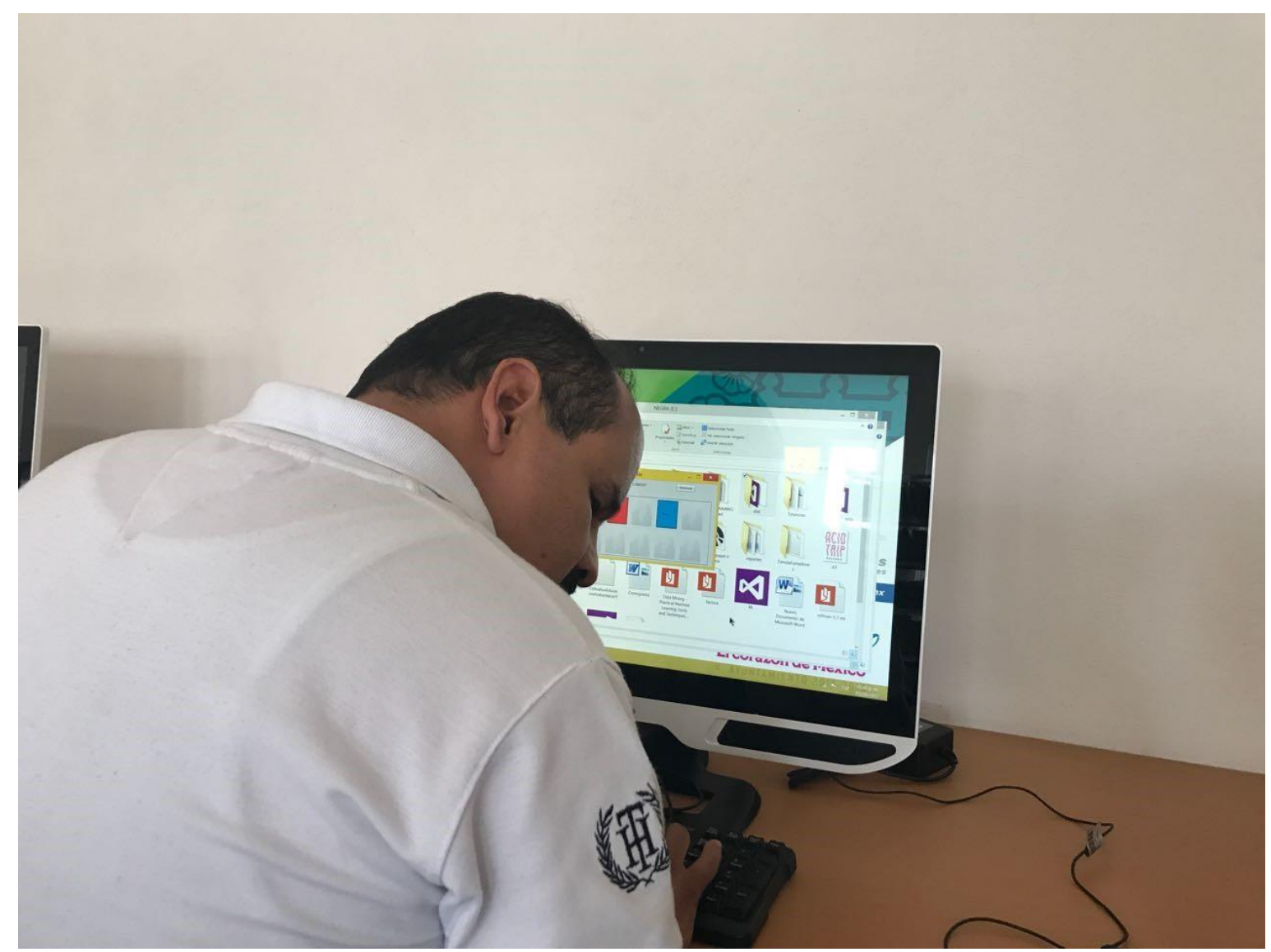

Fig. 5. Pruebas realizadas utilizando la aplicación "Conociendo mi ciudad”, por una persona ciega. Fuente: autores.

\section{CONCLUSIÓN}

La aplicación desarrollada se encuentra en un nivel de interacción aceptable para los usuarios ciegos, sin embargo, existen todavía aspectos a considerar para mejorar la calidad de la aplicación, por ejemplo, una persona que se encuentra en la etapa de pérdida de vista puede no ser atractivamente visual. Por otro lado, la funcionalidad de la aplicación es correcta con base a los requerimientos de la aplicación, ya que ésta da a conocer los puntos importantes de la ciudad de Aguascalientes, México, y la historia del lugar, por lo que el usuario puede empezar a reconocer los lugares importantes que tiene Aguascalientes, memorizar y facilitar la movilidad del usuario.

Como trabajo a futuro: se desarrollará una siguiente versión, la cual contendrá los lugares típicos de comida donde tiene tu- rismo la ciudad y, además, con base en las observaciones realizadas por personas que se encuentran en un nivel de discapacidad de pérdida de vista es necesario mejorar la apariencia para no solo hacer la aplicación para personas ciegas y considerar usuarios que se encuentran en un nivel avanzado de pérdida de vista; cabe destacar que para personas ciegas cumple el objetivo de ser un sistema interactivo y les ayuda en el desarrollo personal.

\section{REFERENCIAS}

[1] H. Schrott, "Diseñar para los discapacitados," Rev. la OMPI, no. 5, p. 32, 2009.

[2] W. S. Humphrey, Introduction to the team software process. Addison-Wesley Professional, 1999.

[3] O. M. de la Salud, "Discapacidad y salud," Organización Mundial de la Salud, 2016. [Online].

Available: 
http://www.who.int/mediacentre/factsheets/fs 352/es/. [Accessed: 30-Apr-2016].

[4] R. A. Koon and M. E. de la Vega, "El impacto tecnológico en las personas con discapacidad," in II Congreso Iberoamericano de Informática Educativa Especial, 2014, p. 19.

[5] INEGI, "Discapacidad en México," Cuéntame, 2014. [Online]. Available: http://cuentame.inegi.org.mx/poblacion/disca pacidad.aspx?tema=P. [Accessed: 17-Oct2017].

[6] Unicef and MINEDUC, Inclusión social, discapacidad y políticas públicas. 2004.

[7] J. A. Ferreyra, A. Méndez, and M. A. Rodrigo, "El uso de las TIC en la Educación Especial: Descripción de un sistema informático para niños discapacitados visuales en etapa preescolar," Rev. Iberoam. Tecnol. en Educ. y Educ. en Tecnol., no. 3, pp. 55-62, 2009.

[8] J. Drummond, "Understanding Interactive Systems," Organised Sound, vol. 14, no. 2, p. 124, Aug. 2009.

[9] I. Sommerville, Ingeniería del software. Pearson Educación de México, S.A. de C.V., 2011.

[10] H. Oktaba, F. García, M. Piattini, F. Ruiz, F.
J. Pino, and C. Alquicira, "Software Process Improvement: The Competisoft Project," Computer (Long. Beach. Calif),, vol. 40, no. 10, pp. 21-28, Oct. 2007.

[11] R. Pressman, Ingeniería de Software. Un enfoque práctico, 7th ed. Mc Graw Hill, 2010.

[12] F. Auna, Las personas con discapacidad frente a las tecnologías de la información y las comunicaciones en España. Madrid: Fundación Auna-Ministerio de Trabajo de Asuntos Sociales, 2003

[13] J. Muñoz, C. Hernandez, V. Bustos, M. Calderon, C. Collazos, and Y. Méndez, Temas de Diseño en Interacción HumanoComputadora, 1st ed. Iniciativa Latinoamericana de Libros de Texto abiertos (LATIn), 2014.

[14] T. Álvarez, A. García, and A. Sánchez, "Hacia una metodología de interfaz móvil basada en audio para recorridos en interiores como apoyo a personas con discapacidad visual," en Educação Especial na Perspectiva da Educação Inclusiva: concepções e práticas educativas, ISBN 978-85-99643-37-2, pp. 167-176, 2016.

[15] J. Nielse, Usability Engineering. San Diego: Elsevier, 1994. 